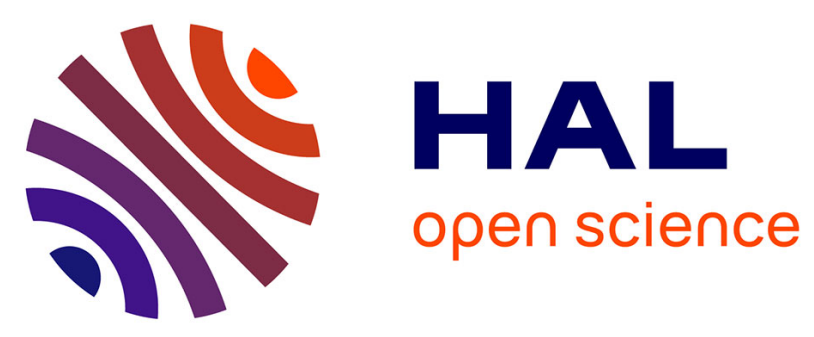

\title{
Mapping Transport Properties of Halide Perovskites via Short-Time-Dynamics Scaling Laws and Subnanosecond-Time-Resolution Imaging
}

Guillaume Vidon, Stefania Cacovich, Marie Legrand, Armelle Yaiche, Daniel Ory, Daniel Suchet, Jean-Baptiste Puel, Jean-François Guillemoles

\section{To cite this version:}

Guillaume Vidon, Stefania Cacovich, Marie Legrand, Armelle Yaiche, Daniel Ory, et al.. Mapping Transport Properties of Halide Perovskites via Short-Time-Dynamics Scaling Laws and Subnanosecond-Time-Resolution Imaging. Physical Review Applied, 2021, 16 (4), 10.1103/PhysRevApplied.16.044058 . hal-03474229

\author{
HAL Id: hal-03474229 \\ https://hal.science/hal-03474229
}

Submitted on 10 Dec 2021

HAL is a multi-disciplinary open access archive for the deposit and dissemination of scientific research documents, whether they are published or not. The documents may come from teaching and research institutions in France or abroad, or from public or private research centers.
L'archive ouverte pluridisciplinaire HAL, est destinée au dépôt et à la diffusion de documents scientifiques de niveau recherche, publiés ou non, émanant des établissements d'enseignement et de recherche français ou étrangers, des laboratoires publics ou privés. 


\section{Mapping transport properties of halide perovskites via short time dynamics scaling laws and sub-nanosecond time resolution imaging}

Guillaume Vidon, ${ }^{1,2, *}$ Stefania Cacovich, ${ }^{1,2}$ Marie Legrand,,${ }^{1,3}$ Armelle Yaiche, ${ }^{1,3}$ Daniel Ory, ${ }^{1,3}$ Daniel Suchet, ${ }^{1,2}$ Jean-Baptiste Puel, ${ }^{1,3}$ and Jean-François Guillemoles ${ }^{1,2, \dagger}$

${ }^{1}$ IPVF, Institut Photovoltaïque d'Ile-de-France, 18 Boulevard Thomas Gobert, 91120 PALAISEAU, France

${ }^{2}$ Institut Photovoltaïque d'Ile de France, UMR IPVF 9006, CNRS, Ecole Polytechnique, Institut Polytechnique de Paris, PSL Chimie ParisTech, IPVF SAS, 91120 PALAISEAU, France

${ }^{3}$ EDF RED, 30 RD 128, 91120 PALAISEAU, France

(Dated: December 10, 2021) 


\begin{abstract}
The excellent optoelectronic and transport properties of halide perovskites led to a rapid development of perovskite based optoelectronic devices. The fundamental understanding of charge carrier dynamics as well as the implementation of physical models able to accurately describe their behaviour are essential for further improvements in the field. Here, combining advanced modeling and characterization, a method for analyzing the short time dynamics of time resolved fluorescence imaging (TR-FLIM) decays is demonstrated. A theoretical scaling law for the time derivative of transient photoluminescence decays as a function of the excitation power is extracted. This scaling law, computed from classical drift-diffusion equations, defines an innovative and simple way to extract quantitative values for several transport parameters including the external radiative recombination coefficient. The model was notably applied on a set of images acquired with a temporal shift of $250 \mathrm{ps}$ to map the top surface recombination velocity of a triple cation mixed halide perovskite thin film at the microscale. The development of high-time-resolution imaging techniques coupled with the scaling method for analyzing short-time dynamics provides a solid platform for the investigation of local heterogeneities in semiconductor materials and the accurate determination of the main parameters governing their carrier transport.
\end{abstract}

\title{
I. INTRODUCTION
}

Over the last decade, lead-halide perovskites solar cells have proved to be a serious candidate for reaching large scale photovoltaic (PV) solar energy conversion, a much-needed solution to meet climate targets and move towards a low-carbon economy. However, despite this emerging technology has recently reached a record power conversion efficiency of $25.5 \%$ for a single junction device $[1,2]$, several open questions persist in the scientific community, ranging from the nature and densities of the defects in the absorbers [3-5], the optimal design for the interfaces energetics in a full device [6, 7], and the long term stability [7-9].

The optimization of device performances goes along with the complete understanding of complex recombination processes occurring both in the bulk and at the interfaces. Due to the interplay of several chemical and physical parameters in these hybrid compounds, such

\footnotetext{
* guillaume.vidon@ipvf.fr

$\dagger$ jean-francois.guillemoles@cnrs.fr
} 
studies often require the use of advanced characterization methods. Among those, time resolved photoluminescence (TR-PL) is widely used to probe halide perovskites transport properties $[10,11]$. If the TR-PL experiments are rather easy to perform, difficulties lie in the correct physical interpretation of the signal $[12,13]$. To date, two main approaches are employed in the literature: one based on phenomenological fitting of the decays and the other on the use of drift-diffusion equations. In the first case, fitting functions such as mono-exponential, bi-exponential [14], stretched exponential [7, 15, 16], tri-exponential [17] are arbitrarily chosen. Though fitting the decays with these custom chosen functions often yields close correspondence with the observed signal, the fitted time constants and parameters have no strict physical meaning. The second method relies on a microscopic drift-diffusion model to describe the dynamics of carriers transport and recombination [1823]. This approach has also been employed as the basis for the development of more complex models, aimed at investigating in-depth diffusion $[19,24]$ or photon recycling $[25,26]$. However, this interpretation method has multiple drawbacks. First of all, these models assess a large number of microscopic parameters from the fit of a handful of decays, such as diffusion coefficient, recombination rate constants, interface properties, device geometry. Therefore, questions arise on the number of independent parameters that can be determined with this method. Another major drawback stems from the fact that multiple sets of parameters can account satisfyingly for the same measured signal [27]. Moreover, uncertainties computations are not often detailed and the correlation between fitted parameters are rarely reported. Another important issue concerns the treatment of large datasets acquired by time resolved imaging set-ups, such as time-resolved fluorescence imaging (TR-FLIM). Indeed, fitting decays with drift diffusion models can be time-consuming, therefore performing fits for every pixel of an image requires intense computational power. Thus, to accurately map local transport parameters, new approaches in modeling as well as data treatment are required.

In this study, we established a theoretical relationship that allows for the direct determination of physical parameters without using drift-diffusion fitting. This method directly outputs coefficients that are related to physical parameters via theoretical formulae. Our approach is based on a scaling law we derived for the time-derivative of the transient PL signal computed just after the laser pulse. Other groups used the derivative of the PL signal to describe different physical phenomena. For instance, Krogmeier and co-workers [28] 
defined a differential lifetime of halide perovskite closely linked with such derivative [18]. Saba and co-workers displayed the initial derivative as a function of laser fluence studied on $\mathrm{MAPbI}_{3}$ and $\mathrm{MAPbI}_{3-x} \mathrm{Cl}_{x}$ perovskite [29]. However, only in few publications [21, 30] were the theoretical components of the PL derivative directly associated to their physical origin, i.e. carrier diffusion, recombination in the bulk or at the interfaces via traps and radiative recombination. In these publications, the derivative is computed in a general and thus integral form. As no specific analytical form for the inhomogeneous photo-generated carrier density profile $\Delta n(z, t)$ is considered, the integrals are not computed.

We propose to compute the expected derivative and integrals at 'short - time' after the pulse, when one can make an assumption on the photo-generated carrier density spatial profile based on Beer-Lambert's law. We obtained a law that is linear in laser fluence. Starting from this law and from the observation of the short time derivative of the PL signal, it is possible to extract accurate values of physical parameters such as the external radiative recombination coefficient or top surface recombination velocity. This method can also be applied for the analysis of three-dimensional datasets, acquired with both imaging systems and confocal PL setups, although very precise signal is needed for local application. One of the benefits of considering only the beginning of the decays stands in the high signalto-noise ratio of the curves in this temporal window.

The process flow of the paper is as follows. We first discuss the theoretical approach we used to derive the scaling law at short-time. Then, we experimentally validate the model by determining the main transport parameters of a state-of-the-art triple-cation lead halide perovskite thin film. Using this dataset, we show that this technique allows to accurately map transport properties such as the top surface recombination velocity. Finally, we comment on the range of validity of the technique as well as on the quality of its output parameter values, and compare this approach to the usual drift-diffusion fitting technique to highlight their complementarity. 


\section{MODEL DESCRIPTION}

\section{A. The Drift-Diffusion Model}

In this section, we introduce the theoretical concepts leading to the scaling law we established. The first hypothesis we make is to tackle the case of intrinsic materials. We consider a slab of intrinsic semiconductor material of thickness $L$. As the material is intrinsic, and as the electric field is not considered, we assume that the electron and hole local densities are equal at any time and position, meaning $n_{e}(z, t)=n_{h}(z, t) \equiv n(z, t)$. The sample is illuminated by a pulsed laser from its top surface. The pulse duration of the laser later used in this study is of the order of tens of picoseconds. However, the time resolution of the gates of the TR-FLIM set-up is of the order of nanoseconds, therefore we model the pulse as an initial condition for the excess carrier density $\Delta n(z, t)$ and not as a time dependent generation function. This kind of approximation has been previously used in the literature, for instance by Maiberg and co-workers [21]. For the initial spatial profile of the excess carrier density, we assume Beer-Lambert's law:

$$
\Delta n(z, t=0)=n_{\gamma} \alpha e^{-\alpha z}
$$

with $n_{\gamma}$ the fluence of the laser in ph.cm ${ }^{-2}$ per pulse and $\alpha$ in $\mathrm{cm}^{-1}$ the absorption coefficient of the material at the excitation wavelength $\lambda$ of the laser.

From this initial state, the system will relax towards the equilibrium solution $\Delta n=0$. The drift-diffusion equation is used to describe this relaxation. We write it in the following form, valid at each position $z$ inside the absorber and time $t>0$ [31]:

$$
\frac{\partial \Delta n}{\partial t}(z, t)=D \frac{\partial^{2} \Delta n}{\partial z^{2}}(z, t)-R(z, t)
$$

with $D$ the diffusion coefficient in $\mathrm{cm}^{2} \mathrm{~s}^{-1}$, and $R(z, t)$ the recombination rate in $\mathrm{cm}^{-3} \mathrm{~s}^{-1}$ at position $z$ and time $t$. We consider two main types of bulk recombination: recombination via traps, or Shockley-Read-Hall (SRH) recombination [32, 33], and radiative recombination. The recombination rate is therefore:

$$
R=k_{1} \frac{(\Delta n)^{2}}{\Delta n+N_{b u l k}}+k_{2}(\Delta n)^{2}
$$

where we introduced the non-radiative recombination rate coefficient $k_{1}$ in $\mathrm{s}^{-1}$ and the external radiative recombination rate coefficient $k_{2}$ in $\mathrm{cm}^{3} \mathrm{~s}^{-1} . N_{b u l k}$ accounts for the case 
of defects that have an energy close to one of the bands, see more details in Supplemental Material [34] Section 1.1. Note that, in Equation 3, the intrinsic carrier density $n_{i}$ was neglected with respect to $\Delta n$ leading us to approximate $n^{2}-n_{i}^{2} \approx \Delta n^{2}$.

While photon recycling was shown to play an important role for thick perovskite absorbers $[35,36]$, the impact of this process on thin-films is still under debate. One way to take photon recycling into account is [37] by introducing a factor applied to the internal radiative recombination coefficient, namely $k_{2}=p_{\text {esc }} k_{2}^{i n t}$, with $p_{\text {esc }}<1$ being the probability of escape for a photon, and $k_{2}^{\text {int }}$ the internal radiative recombination coefficient. A more advanced way of modeling was proposed by Ansari-Rad and Bisquert who considered an effective drift-diffusion equation for the photons coupled with the ones of the carriers [38]. We used their model to evaluate the impact of photon recycling and light outcoupling in the case under study. The details are presented in the Supplemental Material section I.E. The main conclusion is that photon-recycling plays close to no role in the present case because we consider thin layers compared to the absorption length at the emission wavelength. It could however in other cases have an impact by modifying the observed external radiative recombination coefficient. We therefore leave $k_{2}$ to represent the external radiative recombination coefficient for a general model, even though our experimental conditions correspond to a case where it close to the internal radiative recombination coefficient.

Auger recombination was not considered as it is relevant only at very high carrier densities $>10^{17} \mathrm{~cm}^{-3}$, at least for $\mathrm{MAPbI}_{3}$ perovskites [39]. However, as detailed in the Supplemental Materials section I.D, Auger terms can be taken into account. Our observations conclude that they are negligible in the case under study.

From the definition of the radiative recombination rate, we can set the PL intensity received by the detector at time $t$ as:

$$
P L(t)=A \times \int_{0}^{L} \Delta n^{2}\left(z^{\prime}, t\right) d z^{\prime}
$$

with $A$ being a proportionality factor that describes the precise losses and geometry of the measurement setup.

Recombination processes occurring at the interfaces have been proved to critically affect device performance $[40,41]$. We thus introduce in the model surface recombination, at the top and bottom interfaces. At the top interface,

$$
D \frac{\partial \Delta n}{\partial z}(z=0, t)=S_{t o p} \frac{\Delta n(z=0, t)^{2}}{\Delta n(z=0, t)+N_{t o p}}
$$


where $S_{\text {top }}$ is the top surface recombination velocity, and $N_{\text {top }}$ is the corresponding defect related density, similar to $N_{\text {bulk }}$. A similar expression (with a minus sign) is considered at the bottom interface at $z=L$ with $S_{\text {bot }}$ being the bottom surface recombination velocity and $N_{\text {bot }}$ the corresponding defect density.

\section{B. The scaling law at short time after the pulse}

Guided by the fact that all driving forces are present at initial time, we compute analytically the time-derivative shortly after the pulse of the PL signal normalized by its maximum value:

$$
\left.\frac{d P L_{r}}{d t}\right|_{t=0}=\frac{1}{P L(t=0)} \times\left.\frac{d P L}{d t}\right|_{t=0}
$$

To go further, we make the following and main approximation. We assume that, shortly after the pulse, the carrier density is both close to its initial Beer-Lambert-like state and all the while respecting the drift diffusion equation. Using this approximation, along with the definition of the PL (Equation 4) and the drift-diffusion equation (Equation 2), one can compute the theoretical value of Equation 6. A general form can be computed, as demonstrated in Supplemental Material Section 1.2. This extended form can be further simplified with additional assumptions: (i) that the excitation wavelength is strongly absorbed $\left(e^{-\alpha L} \ll 1\right)$ and (ii) that the defect related densities are negligible compared to the excitation densities $\left(n_{\gamma} \alpha \gg N_{b u l k}, N_{t o p}\right)$. More details on the approximation validity are given in the Supplemental Materials section I.B. Under such approximations we obtain the following scaling law:

$$
\left.\frac{d P L_{r}}{d t}\right|_{t=0}=-2\left[k_{1}+\alpha^{2} D+2 \alpha S_{\text {top }}\right]-\frac{4}{3}\left[k_{2} \alpha\right] n_{\gamma}
$$

This equation relates the derivative of the normalized decay curve just after the laser pulse to the model parameters, notably to the experimentally accessible parameters: the fluence $n_{\gamma}$ and the laser-wavelength-dependent absorption coefficient $\alpha(\lambda)$. The slope of this linear behavior gives a direct access to the product $\alpha k_{2}$. The absorption coefficient can be measured via other methods such as Fourier transform photocurrent spectroscopy [42, 43] or ellipsometry [44]. Therefore, one can extract a direct measurement of the external radiative recombination rate $k_{2}$. The intercept of the scaling gives a direct access to the quantity $k_{1}+\alpha^{2} D+2 \alpha S_{t o p}$. There is a major difference between the intercept of the scaling at short 
time and the (long-time) observed lifetime: the spatial distribution of the carriers. For the short-time intercept it follows Beer-Lambert's law, while for the lifetime a more homogeneous distribution is reached (not entirely homogeneous due to surface recombination). These two distributions explain the difference in terms of theoretical formulae between these two characteristic times of the system.

Equation 7 suggests two sets of experiments. The first one consists in measuring decays when varying the laser fluence at a given laser wavelength. This approach has been widely employed in the literature $[20,22,24,28]$. In this case, one could explore how the initial slopes of the relative decays exhibit a linear relationship with laser fluence. The other set of experiments would consist in varying the laser wavelength at fixed laser fluence. Then, as long as the high absorption condition $e^{-\alpha L} \ll 1$ is fulfilled, one expects a polynomial of order 2 in $\alpha$ for the initial derivative (a generalized version of the scaling for the low absorption case is given in Supplemental Materials Section I.F). Combining the two methods would give access to most of the model parameters: $k_{1}, D, S_{t o p}, k_{2}$, without any use of complex numerical models. This second set of experiments will be the subject of a future study, while we will tackle fluence-dependent studies in the rest of this paper.

\section{EXPERIMENTAL RESULTS ON TRIPLE CATION PEROVSKITE}

To validate the proposed model, we performed TR-FLIM experiments on triple cation perovskite thin films $\left(\mathrm{Cs}_{0.05}\left(\mathrm{MA}_{0.17} \mathrm{FA}_{0.83}\right)_{0.95} \mathrm{~Pb}\left(\mathrm{Br}_{0.17} \mathrm{I}_{0.83}\right)_{3}\right)$ deposited on glass substrates. The acquisitions were carried out by applying a wide-field pulsed illumination $(\lambda=532 \mathrm{~nm})$ in a protective $N_{2}$ atmosphere at the temperature of $20^{\circ} \mathrm{C}$ to prevent light soaking effects [45]. Each acquisition was repeated three times and good reproducibility was obtained as

reported in Supplemental Material Figure 2. We acquired images with a 3ns-long exposure gate. To further improve the time resolution of our measurement, we acquired one image every 250 ps during 19.5 ns, see Figure 1 (a). Thus, we obtained sub-nanosecond-resolved images and decays. One of the advantages of the sub-nanosecond-resolved curves is that they allow to verify that the maximum of the PL decay is not missed by a de-synchronisation between the laser pulse and the gated-camera - which is critical when normalizing decays. However, for such temporal resolution, it is necessary to consider the convolution of the signal with the camera's temporal response function. We also acquired regular data cubes, 

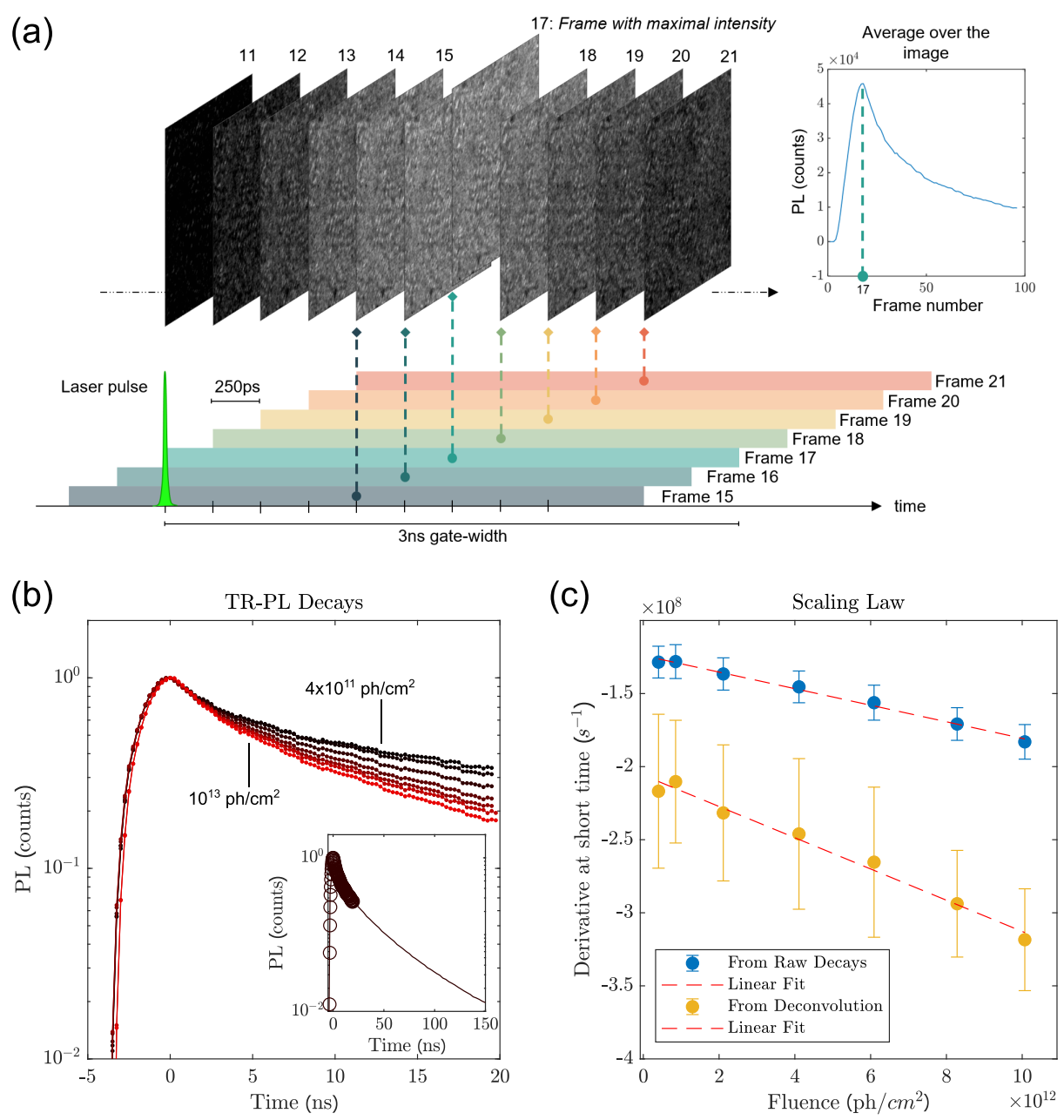

FIG. 1. Experiments on triple-cation perovskite $\left(\mathrm{Cs}_{0.05}\left(\mathrm{MA}_{0.17} \mathrm{FA}_{0.83}\right)_{0.95} \mathrm{~Pb}\left(\mathrm{Br}_{0.17} \mathrm{I}_{0.83}\right)_{3}\right)$ thin films in inert atmosphere. (a). Scheme of the sub-nanosecond-resolved TR-FLIM acquisitions. The 3 ns-long gates are slided in time with an interval of 250 ps. For each gate, an image is acquired. The temporal convolution of the signal with the gates results in a delay between the laser pulse (a few tens of picosecond long) and the center of the maximal frame. Top right is the obtained decay averaged over the images. All images share the same colorscale (from $3.5 \times 10^{4}$ to $6 \times 10^{4}$ counts). (b). TR-PL intensity, computed as the space integration of the TR-FLIM signal, as a function of time for different fluences acquired with a time shift of 250 ps. Dark curves correspond to lower fluences (down to $4 \times 10^{11}$ ph.cm ${ }^{-2}$ ) and red curves to higher fluences (up to $10^{13}$ ph.cm ${ }^{-2}$ ). (b-Inset) Comparison of the sub-nanosecond-resolved curves (open circles) with the regular decays resolved at $3 \mathrm{~ns}$ (line). Only the first $150 \mathrm{~ns}$ of the regular and longer decay (that lasts up to $t=879 \mathrm{~ns}$ ) are shown. (c). Relative slope fitted on the decays between $0.5 \mathrm{~ns}$ and $2.5 \mathrm{~ns}$ as a function of laser fluence: (blue) measured on the raw decays; (yellow) measured on the deconvoluted decays. Error bars are given by the fitting function, see Supplemental Material Section 2 on numerical uncertainty. The curves were fitted by using a linear function (dotted line): 
by taking one image every $3 \mathrm{~ns}$ from $t=-21 \mathrm{~ns}$ up to $t=879 \mathrm{~ns}$, as shown in the inset of Figure $1(\mathrm{~b})$.

In Figure 1 (b), we plotted sub-nanosecond-resolved decays acquired at different laser fluences (varying from $4 \times 10^{11}$ to $1 \times 10^{13}$ ph.cm ${ }^{-2}$ ) and averaged over the whole image. The corresponding scaling is shown in Figure 1 (c), in blue dots. The derivative was computed as the slope of a linear fit of the natural logarithm of the PL between 0.5 and 2.5ns. Two data sets are considered: in the former the derivative was performed on the raw decays shown in Figure 1 (b) while in latter we computed the derivative on the results of a deconvolution algorithm applied to the decays. The deconvolution algorithm uses Wiener filter and is described in Supplemental Material Section 3, along with the resulting decays. To correctly evaluate the deconvolution, we measured our apparatus response function and used Fast Fourier Transform algorithms to recover the original signal. A parameter, noted $K$ is needed for Wiener filter deconvolution, and taken here to be $K=0.1$. Its influence is discussed in Supplemental Material Section 3. We also tested a third method of deconvolution, via an effective fit of a convolved bi-exponential with results close to the Wiener deconvolution. Details are given in the Supplemental Materials. For both scalings, a linear behaviour for the initial derivative with the laser fluence is observed, as expected from Equation 7. Then, we fit linearly the points of Figure 1 (c), to obtain the slope $a=-4 / 3 \alpha k_{2}$ and intercept $b=-2\left[k_{1}+\alpha^{2} D+2 \alpha S_{t o p}\right]$ of the scaling law. For the raw dataset, we obtained values of $a=-(5.7 \pm 0.6) \times 10^{-6} \mathrm{~cm}^{2} \mathrm{~s}^{-1}$ and $b=-(1.24 \pm 0.03) \times 10^{8} \mathrm{~s}^{-1}$, whereas for deconvoluted dataset we find $a=-(1.07 \pm 0.15) \times 10^{-5} \mathrm{~cm}^{2} \mathrm{~s}^{-1}$ and $b=-(2.06 \pm 0.08) \times 10^{8} \mathrm{~s}^{-1}$.

We can make three remarks on the impact of temporal convolution. One, the order of magnitude of the parameters is similar for both results after and before deconvolution. Two, characterizing the apparatus response function allows for the use of deconvolution algorithms. These algorithms are especially efficient in the high signal to noise regions, which is exactly our temporal window of interest when computing the short-time derivative. Three, the use of deconvolution algorithms is necessary because the characteristic timescales of the material's decay are comparable to the temporal resolution of our camera. We want to stress that deconvolution would not be necessary for the study of materials with longer characteristic time scales.

Furthermore, we use the results from the deconvoluted dataset $\left(k_{2} \alpha_{532 \mathrm{~nm}}=(8.0 \pm 1.1) \times\right.$ $\left.10^{-6} \mathrm{~cm}^{2} \mathrm{~s}^{-1}\right)$ to estimate the transport parameters of the perovskite layer. Assuming an 
absorption coefficient at the laser wavelength $(532 \mathrm{~nm}) \alpha=(6.5 \pm 1) \times 10^{4} \mathrm{~cm}^{-1}$ from previous measurements, and in line with literature values for this composition and laser wavelength [35], we can extract the value of the external radiative recombination coefficient $k_{2}=(1.2 \pm 0.4) \times 10^{-10} \mathrm{~cm}^{3} \mathrm{~s}^{-1}$ by using Equation 7 . Moreover, we can provide lower and upper bounds for the multiple parameters expressed in the intercept coefficient. The bulk lifetime $\tau=1 / k_{1}$ would in this regard be such that $\tau \geq 10 \mathrm{~ns}$, which is highly expected for triple cation perovskites. The diffusion coefficient would be less than $D \leq 2.4 \times 10^{-2} \mathrm{~cm}^{2} \mathrm{~s}^{-1}$, corresponding to a mobility $\mu \leq 0.9 \mathrm{~cm}^{2} \mathrm{~s}^{-1} \mathrm{~V}^{-1}$ at ambient temperature. For the top surface recombination velocity, one finds that $S_{t o p} \leq 790 \mathrm{~cm} . \mathrm{s}^{-1}$. All of these conditions are in agreement with literature results on triple cation perovskites [35], and with our previous results [24].

This analysis can also be performed locally on images, as shown in Supplemental Material Section IV and Figure S8. For each pixel, a binning with the neighbouring pixels was performed (10x10pix, centered) and a linear scaling was fitted from the derivatives measured on the local deconvoluted decays. The maps of the slope and intercept of the scalings are displayed in Supplemental Material Figure S8 (a) and (d). We can observe local variations that are negatively correlated between the intercept and the slope, see Supplemental Material Fig S8 (c). According to our simulations, this anti-correlation can be explained by noise in the determined derivative of the decays, see Supplemental Material Fig S8 (f). This means that local application of the scaling law suffers from high noise on the local decays in our data-set. With such level of local noise, we are unable to output independent maps of slope and intercept, which would lead to independent maps of material parameters.

However, one advantage of our approach over phenomenological fitting is that it allows to make hypothesis based on physical considerations. In the situation currently under scrutiny, we can assume that the bulk properties of the film are uniform, and that heterogeneities are caused by local differences in the local top surface recombination velocity. Under this hypothesis all local scalings should have the same slope, since the absorption coefficient $\alpha$ and the external radiative recombination coefficient $k_{2}$ are considered constant. We evaluated the impact of the local outcoupling on the slope of scaling via a modification of the external radiative recombination coefficient and found it to be less than the sensitivity of our technique - moreover the outcoupling was found to have no impact on the intercept of the scaling. Therefore, under this approximation, only the intercept of the scalings should vary 
locally, and its variation is attributed to a variation in local surface recombination velocity, see Equation 7. We fix the slope of local scalings to the value we obtained from the spatially averaged decays, $a=-(1.07 \pm 0.15) \times 10^{-5} \mathrm{~cm}^{2} \mathrm{~s}^{-1}$, and compute only the local intercept. Doing so, we obtained a map of the local intercept of the scaling. By assuming values for the bulk SRH recombination parameter $k_{1}$ and the diffusion coefficient $D$, it is possible to extract a map of the local top surface recombination velocity $S_{t}$ as shown in Figure 2 (a). The values we assume for $D$ and $k_{1}$ are the results of a drift-diffusion fit of longer decays presented in the Discussion section. In Figure 2 (b) we show a vertical cross section of the map. We can see variations that are above the estimated error bars. To justify our assumption that local surface recombination is the origin of the variation of the intercept, we evaluate, from Equation 7 and the chosen parameter set, that top surface recombination account for $76 \%$ of the value of the intercept. We can thus conclude that the scaling law, combined with hypothesis on the material under study, allows us to observe local variations of parameters in a direct way.

To test the technique further, we performed experiments on in-homogeneous samples: perovskite on glass that had been exposed to a X-Ray beam. The application of the scaling law technique is shown in Supplemental Materials Section IV.B and Figure S10. In this case, we imaged variations of local intercepts that correspond to the X-Ray spot, while the slope of the scaling showed little variation. In this in-homogeneous case, no hypothesis was required as we found uncorrelated variations of slopes and intercepts.

\section{DISCUSSION}

In this section, we discuss the obtained results. First, we comment on the values of the parameters obtained with this technique. Then, we compare this technique with regular drift-diffusion fitting and we demonstrate that using the scaling law leads to a higher level of accuracy in the determination of parameters. Next, we discuss the validity of the assumptions leading to Equation 7, describing the scaling law. Finally, we discuss the limits of the proposed technique and possible future improvements.

By following the scaling law approach, we obtained a value for the external radiative

recombination coefficient $k_{2}$ equal to $k_{2}=(1.2 \pm 0.4) \times 10^{-10} \mathrm{~cm}^{3} \mathrm{~s}^{-1}$. According to Brenes et al. [35], the internal radiative recombination coefficient of $\mathrm{Cs}_{0.05}\left(\mathrm{MA}_{0.17} \mathrm{FA}_{0.83}\right)_{0.95} \mathrm{~Pb}$ - 
(a)

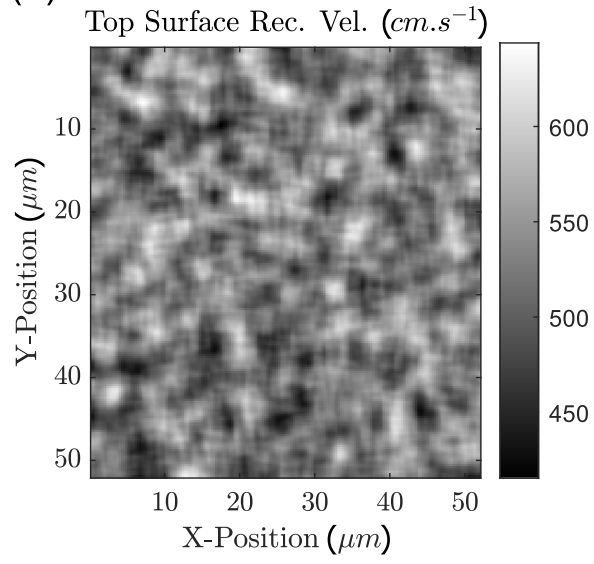

(b)

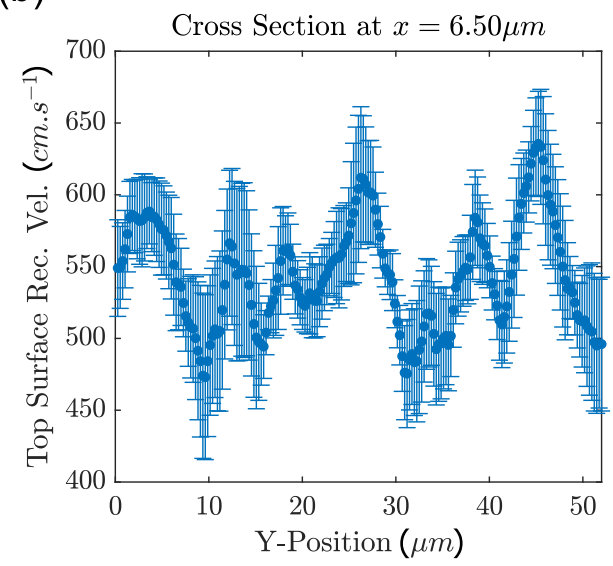

FIG. 2. Local determination of parameters. (a). Map of top surface recombination velocity from the fitted local intercepts of the local scalings. The 7 fluences acquisiton of Figure 1 were used at a local scale. A binning of 10 pixels is applied, meaning that all pixels are averaged out on a square of size $10 \times 10$ pixels. For each pixel, seven decays are obtained, corresponding to seven fluences. The derivative of the deconvoluted decays are computed between 0.5 and $2.5 \mathrm{~ns}$. The derivatives are assembled to form the scaling, from which the intercept is fitted. The slope for all points is set to $a=-1.07 \times 10^{-5} \mathrm{~cm}^{2} \mathrm{~s}^{-1}$. To obtain the map of local intercept, we compute locally the average of the ensemble $E=\{$ derivative $-a \times$ fluence $\}$. For the uncertainty, we compute $\operatorname{std}(E) / \sqrt{\# E}$, with \#E the number of elements in $E$. Then we use Equation 7 and the assumed values for the other parameters to compute the map of $S_{t o p}$ from the map of the intercept. The parameters were estimated at $D=3.54 \times 10^{-3} \mathrm{~cm}^{2} \mathrm{~s}^{-1}, k_{1}=9.23 \times 10^{6} \mathrm{~s}^{-1}, \alpha=6.5 \times 10^{4} \mathrm{~cm}^{-1}$. (b) Vertical cross section of map (a) at $x=6.5 \mu \mathrm{m}$.

$\left(\mathrm{Br}_{0.17} \mathrm{I}_{0.83}\right)_{3}$ perovskite is of the order of $k_{2}=2.0 \times 10^{-10} \mathrm{~cm}^{3} \mathrm{~s}^{-1}$, while the external radiative recombination coefficient, evaluated taking into account photon recycling, is of the order of $k_{2}^{e x t}=1.0 \times 10^{-11} \mathrm{~cm}^{3} \mathrm{~s}^{-1}$. Firstly, we can observe that our technique is able to obtain a value close to what is expected for such materials. Secondly, the value we find is close to the internal radiative recombination coefficient as was expected by our simulations of the impact of photon-recycling in the present case, see Supplementary Section I.D. The difference between the value given in [35] and ours can have different origins. One is the overall uncertainty on this parameter which is still not determined with precision. Two, the obtained value is dependent on the measure of the absorption coefficient at the laser wavelength $\alpha$. Three, we showed that the temporal convolution has an impact on the 
determination of the radiative coefficient. This calls for a more detailed study on the best method to deconvolute the signal and obtain the most precise estimate. Therefore, we conclude that the scaling law allows to extract a direct value for the external radiative recombination coefficient which, in the present low photon-recycling case, is close to the internal radiative recombination coefficient.

In order to ascertain the accuracy of the parameters we obtain from this measurement technique, we performed a comparison with the drift-diffusion fit of longer decays. The parameter that our scaling was able to determine is the radiative recombination coefficient $k_{2}$. Therefore, we implemented two fitting strategies. One in which $k_{2}$ is used as a fitting parameter, named Strategy 1, and one where we fixed $k_{2}$ to the value obtained by the scaling measurement, named Strategy 2. For both, we also fit $D, k_{1}, N_{b u l k}, S_{t}, N_{t o p}$. We fitted the 7 TR-PL curves all together at once and with only one model for all the curves: no parameter is changed as a function of fluence. Results of the first fit are presented in Supplemental Material Figure 6. For Strategy 2, the fitted parameters are available in Supplemental Material Table 1 while the correlations between fitted parameters are given in Supplemental Material Table 2. Overall, the correlations are reasonably low. The highest correlations are between $D, N_{\text {top }}$ and $N_{\text {bulk }}$, and equal +0.78 , indicating that these three parameters have a similar bending effect on the decay curves.

We compared the results of the two fitting strategies: comparable values for the fitted parameters were obtained in the two cases. In addition, the uncertainties on the fitted parameters were reduced when considering the value of $k_{2}$ determined by the scaling (see Supplemental Material Table 3). In particular, the uncertainty on $N_{\text {top }}$ goes from $42 \%$ when $k_{2}$ is a free fitting parameter to $25 \%$ when $k_{2}$ is fixed. Finally, the value of the radiative recombination from the drift-diffusion fit is $k_{2}^{d d}=(1.16 \pm 0.07) \times 10^{-10}$, which is fully compatible with the value obtained with the scaling. Therefore we conclude that the scaling law is complementary to regular drift-diffusion fitting.

Moreover, the experimental observation of the linear scaling proves that our assumptions to obtain it were reasonable. Namely, four main assumptions led to Equation 7: (i) the intrinsic nature of the perovskite material, which, according to this experiment, is doped less than $3 \times 10^{16} \mathrm{~cm}^{3}$; In a recent study [46] by Feldmann et al. photodoping of the perovskite was evidenced at time scales of the order of tens of nanoseconds. Our technique is not sensitive to such effects as it relies on the very first nanoseconds of the decays ; (ii) 
at short-time, in our experimental conditions, one can neglect the defect related densities $N_{\text {bulk }}$ and $N_{\text {top }}$ in front of the photo-generated carrier density - which is also confirmed by the values found for these parameters by the drift-diffusion fitting presented above; (iii) no

high order Auger-like recombination were observed, as they would lead to a term $\propto n_{\gamma}^{2}$ in the scaling (see the formula of the scaling in this case and a more detailed discussion in Supplementary Materials); (iv) at short time, the spatial profile of the carriers is both close to Beer-Lambert initial condition and following the drift diffusion equation. Short-time is therefore defined as the time during which this approximation is valid.

However, we also note that our technique has two main experimental limitations. The first one, is due to the camera's temporal response function. To reduce the gap between experimental measurements and theoretical models, we used deconvolution algorithms, as previously discussed in the paper. It is worth noting that this limitation is not due to our method but it is a common issue to all time-convoluted signals. The second one is related to the use of the scaling law approach to map transport parameters. We observed that noise on the local decays creates a negative correlation between the intercept and the slope fitted at a local level. This renders difficult the determination of the two parameters independently at the local scale when the variations on the sample are of the order of the sensitivity of the technique. However, the correlation could potentially also occur for physical reasons, but, to this date, our measurements cannot distinguish the two. This limitation can be surpassed when hypothesis are made on the nature of the material under study, or when the spatial variations are greater than the sensitivity.

\section{CONCLUSION}

We presented a method for acquiring and interpreting TR-PL (or TR-FLIM) data from the combination of advanced modeling and characterization on intrinsic materials. This method relies on the observation of the derivative of the PL decay at short-time. The derivative is analyzed as a function of the laser fluence, with an expected linear behavior. This scaling was obtained from a simple analytical model and applied on triple cation leadhalide perovskites with TR-FLIM measurements to investigate its transport properties. A value of the external radiative recombination coefficient was extracted and then confirmed by a single drift-diffusion model on seven different excitation powers. Moreover, the scaling 
allowed for the local determination of the top surface recombination velocity under the hypothesis of spatially constant bulk properties of the thin film. Most importantly, this approach represents an important step towards more direct measurements of various physical parameters. This is crucial for the determination of the transport properties of perovskite materials, which are still under intense debate in the community. Moreover, the scaling law technique improves classical drift-diffusion fitting, as it allows to reduce the uncertainties on the fitted parameter.

As previously mentioned in the paper, another set of experiments can be performed by varying the laser wavelength. This would lead to the observation of the scaling as a function of the absorption coefficient $\alpha(\lambda)$. Together with the power-study, this excitation-study could allow for the determination of most of the parameters without using a single driftdiffusion code. Finally, the scaling law is valid for probing short-time dynamics but it does not suffice to correctly model phenomena occurring at long-time. Notably, some of the model parameters only matter at a longer time scale. This arouses our interest in the theoretical investigation of the long time-scale dynamics, where complementary physical information is contained.

\section{MATERIAL FABRICATION}

A double cation perovskite solution, $\left(\mathrm{MA}_{0.17} \mathrm{FA}_{0.83}\right) \mathrm{Pb}\left(\mathrm{Br}_{0.17} \mathrm{I}_{0.83}\right)_{3}$ was prepared by dissolving 1.10 $\mathrm{M} \mathrm{PbI}_{2}$ (TCI Chemicals), 0.20 $\mathrm{M} \mathrm{PbBr}_{2}$ (Alfa Aesar), 1.00 M formamidinium iodide (FAI, Dyesol), and 0.20 M methylammonium bromide (MABr, Dyesol) in a mixture of DMSO/DMF (1:4 in v/v) as solvent. In order to obtain the triple cations perovskite, i.e., $\mathrm{Cs}_{0.05}\left(\mathrm{MA}_{0.17} \mathrm{FA}_{0.83}\right)_{0.95} \mathrm{~Pb}-\left(\mathrm{Br}_{0.17} \mathrm{I}_{0.83}\right)_{3}$, Cs + was additionally injected from a precursor solution of CsI (Sigma-Aldrich) 1.50 M in DMSO solvent. The solution, after $2 \mathrm{~h}$ of stirring in a magnetic mixer, was spin-coated onto glass substrates by following a double plateau. First, $35 \mu \mathrm{L}$ of the perovskite solution was spin-coated at the rotation of $2000 \mathrm{rpm}$ for 10 s at an acceleration of $1000 \mathrm{rpm} / \mathrm{sec}$. Then, $100 \mu \mathrm{L}$ of chlorobenzene was spin-coated at a speed of $6000 \mathrm{rpm}$ for $30 \mathrm{~s}$. After deposition, the perovskite films were submitted to an annealing treatment at the temperature of $100{ }^{\circ} \mathrm{C}$ for 30 minutes in a nitrogen glovebox. The thickness of the perovskite thin films was approximately $500 \mathrm{~nm}$. 


\section{EXPERIMENTAL METHODS}

Wide-field illumination imaging was realized with a TR-FLIM setup. The system is composed of a pulsed laser Talisker $(\lambda=532 \mathrm{~nm}, \mathrm{f}=40 \mathrm{kHz})$, an intensified electronmultiplied camera CCD (em-ICCD, PIMAX4, Princeton Instruments), and a home-built opto-mechanical excitation-collection setup. The gate width of the camera was set to $3 \mathrm{~ns}$. For the sub-nanosecond-resolved curves, the camera gating time was varied with a $250 \mathrm{ps}$ step from -5.5 ns to 19.5 ns. For the regular curves, the camera gating time was varied with a 3 ns step from -21 ns to 879 ns. The photoluminescence was collected through a Nikon $50 \times$ high working distance objective with $\mathrm{NA}=0.80$. The sample were placed in a cryostat

chamber set at $20^{\circ} \mathrm{C}$ and filled with nitrogen. The PL was filtered through short-pass and long-pass filters (DMLP650R and FES750, Thorlabs). Each acquisition was repeated three times in a row, with a $5 \mathrm{~s}$ pause in between. Acquisitions were post-treated using Matlab.

\section{SIMULATION METHODS}

Simulations were performed using a home-made Matlab code. The pdepe function is used to solve the drift-diffusion equation on the photo-generated excess carrier density $\Delta n$. This code was validated against Silvaco Atlas simulation tool. For the fits, the convolution of the simulated decays with the apparatus response function is performed with the phenomenological fit of the response function, see the section on deconvolution in the Supplemental Material. For the fitting, all 7 fluences curves are fitted at once, compiled into a 2 dimensional matrix of experimental data. The fitted curves are the natural logarithm of the normalized PL decays. For each curve only the points where normalized PL level is greater than $5 \times 10^{-4}$ were used for the fit.

\section{ACKNOWLEDGEMENTS}

This project has been supported by the French Government in the frame of the program of investment for the future (Programme d'Investissement d'Avenir - ANR-IEED-002-01). S.C. would like to thank funding from the European Union's Horizon 2020 research and innovation programme under the Marie Skłodowoska-Curie Grant Agreement N 845612. 


\section{CONFLICT OF INTEREST}

We declare no conflict of interest.

\section{SUPPLEMENTAL MATERIAL}

Supplemental Material is available at :

[1] G. Kim, H. Min, K. S. Lee, D. Y. Lee, S. M. Yoon, and S. I. Seok, Impact of strain relaxation on performance of a-formamidinium lead iodide perovskite solar cells, Science 370, 108 (2020).

[2] Best Research-Cell Efficiency Chart - Photovoltaic Research - NREL, https://www.nrel. gov/pv/cell-efficiency.html, accessed: 2021-02-18.

[3] H. Jin, E. Debroye, M. Keshavarz, I. G. Scheblykin, M. B. J. Roeffaers, J. Hofkens, and J. A. Steele, It's a trap! On the nature of localised states and charge trapping in lead halide perovskites, Materials Horizons 7, 397 (2020).

[4] S. Reichert, Q. An, Y. W. Woo, A. Walsh, Y. Vaynzof, and C. Deibel, Probing the ionic defect landscape in halide perovskite solar cells, Nature Communications 11, 1 (2020).

[5] S. G. Motti, T. Crothers, R. Yang, Y. Cao, R. Li, M. B. Johnston, J. Wang, and L. M. Herz, Heterogeneous Photon Recycling and Charge Diffusion Enhance Charge Transport in Quasi-2D Lead-Halide Perovskite Films, Nano Lett 19, 11 (2019).

[6] P. Schulz, D. Cahen, and A. Kahn, Halide Perovskites: Is It All about the Interfaces?, Chemical Reviews 119, 3349 (2019).

[7] J. Wang, W. Fu, S. Jariwala, I. Sinha, A. K. Jen, and D. S. Ginger, Reducing Surface Recombination Velocities at the Electrical Contacts Will Improve Perovskite Photovoltaics, ACS Energy Letters 4, 222 (2019).

[8] S. Cacovich, D. Messou, A. Bercegol, S. Béchu, A. Yaiche, H. Shafique, J. Rousset, P. Schulz, M. Bouttemy, and L. Lombez, Light-induced passivation in triple cation mixed halide perovskites: Interplay between transport properties and surface chemistry, ACS Applied Materials and Interfaces 12, $34784(2020)$. 
[9] M. Khenkin, E. Katz, and A. e. a. Abate, Consensus statement for stability assessment and reporting for perovskite photovoltaics based on ISOS procedures, Nature Energy 5, 35 (2020).

[10] G. Delport, S. Macpherson, and S. D. Stranks, Imaging Carrier Transport Properties in Halide Perovskites using Time-Resolved Optical Microscopy, Advanced Energy Materials 10, 1903814 (2020).

[11] W. Chen, Z. Gan, M. A. Green, B. Jia, and X. Wen, Revealing Dynamic Effects of Mobile Ions in Halide Perovskite Solar Cells Using Time-Resolved Microspectroscopy, Small Methods 5, $2000731(2021)$.

[12] E. M. Hutter, T. Kirchartz, B. Ehrler, D. Cahen, and E. v. Hauff, Pitfalls and prospects of optical spectroscopy to characterize perovskite-transport layer interfaces, Applied Physics Letters 116, 100501 (2020).

[13] D. W. Dequilettes, K. Frohna, D. Emin, T. Kirchartz, V. Bulovic, D. S. Ginger, and S. D. Stranks, Charge-Carrier Recombination in Halide Perovskites (2019).

[14] D.-Y. Son, J.-W. Lee, Y. J. Choi, I.-H. Jang, S. Lee, P. J. Yoo, H. Shin, N. Ahn, M. Choi, D. Kim, and N.-G. Park, Self-formed grain boundary healing layer for highly efficient CH3NH3PbI3 perovskite solar cells, Nature Energy 2016 1:7 1, 1 (2016).

[15] D. W. DeQuilettes, S. M. Vorpahl, S. D. Stranks, H. Nagaoka, G. E. Eperon, M. E. Ziffer, H. J. Snaith, and D. S. Ginger, Impact of microstructure on local carrier lifetime in perovskite solar cells, Science 348, 683 (2015).

[16] T. Yamada, Y. Yamada, Y. Nakaike, A. Wakamiya, and Y. Kanemitsu, Photon Emission and Reabsorption Processes in CH3NH3PbBr3 Single Crystals Revealed by Time-Resolved Two-Photon-Excitation Photoluminescence Microscopy, Physical Review Applied 7, 014001 (2017).

[17] M. Zhang, H. Yu, M. Lyu, Q. Wang, J. H. Yun, and L. Wang, Composition-dependent photoluminescence intensity and prolonged recombination lifetime of perovskite $\mathrm{CH} 3 \mathrm{NH} 3 \mathrm{PbBr} 3-\mathrm{x}$ Clxfilms, Chemical Communications 50, 11727 (2014).

[18] R. K. Ahrenkiel and M. Lundstrom, Minority Carriers in III-V Semiconductors: Physics and Applications, Semiconductors and semimetals (Academic Press, Incorporated, 1993).

[19] A. A. Baloch, F. H. Alharbi, G. Grancini, M. I. Hossain, M. K. Nazeeruddin, and N. Tabet, Analysis of Photocarrier Dynamics at Interfaces in Perovskite Solar Cells by Time-Resolved Photoluminescence, Journal of Physical Chemistry C 122, 26805 (2018). 
[20] T. Kirchartz, J. A. Márquez, M. Stolterfoht, and T. Unold, Photoluminescence-Based Characterization of Halide Perovskites for Photovoltaics, Advanced Energy Materials , 1904134 (2020).

[21] M. Maiberg and R. Scheer, Theoretical study of time-resolved luminescence in semiconductors. II. Pulsed excitation, Journal of Applied Physics 116, 123711 (2014).

[22] M. J. Trimpl, A. D. Wright, K. Schutt, L. R. V. Buizza, Z. Wang, M. B. Johnston, H. J. Snaith, P. Müller-Buschbaum, and L. M. Herz, Charge-Carrier Trapping and Radiative Recombination in Metal Halide Perovskite Semiconductors, Advanced Functional Materials 30, $2004312(2020)$.

[23] A. Bercegol, S. Cacovich, G. Vidon, S. Mejaouri, A. Yaiche, J. B. Puel, C. Longeaud, J. F. Guillemoles, S. Jutteau, J. Rousset, D. Ory, and L. Lombez, Imaging Electron, Hole, and Ion Transport in Halide Perovskite, Journal of Physical Chemistry C 124, 11741 (2020).

[24] A. Bercegol, F. J. Ramos, A. Rebai, T. Guillemot, D. Ory, J. Rousset, and L. Lombez, Slow Diffusion and Long Lifetime in Metal Halide Perovskites for Photovoltaics, The Journal of Physical Chemistry C 122, 24570 (2018).

[25] A. Bercegol, D. Ory, D. Suchet, S. Cacovich, O. Fournier, J. Rousset, and L. Lombez, Quantitative optical assessment of photonic and electronic properties in halide perovskite, Nature Communications 10.1038/s41467-019-09527-w (2019).

[26] A. Sridharan, N. K. Noel, B. P. Rand, and S. Kéna-Cohen, Role of Photon Recycling and Band Filling in Halide Perovskite Photoluminescence under Focussed Excitation Conditions, The Journal of Physical Chemistry C 125, 2240 (2021).

[27] T. P. Weiss, B. Bissig, T. Feurer, R. Carron, S. Buecheler, and A. N. Tiwari, Bulk and surface recombination properties in thin film semiconductors with different surface treatments from time-resolved photoluminescence measurements, Scientific Reports 9, 5385 (2019).

[28] B. Krogmeier, F. Staub, D. Grabowski, U. Rau, and T. Kirchartz, Quantitative analysis of the transient photoluminescence of $\mathrm{CH} 3 \mathrm{NH} 3 \mathrm{PbI} 3$ /PC 61 BM heterojunctions by numerical simulations, Sustainable Energy and Fuels 2, 1027 (2018).

[29] M. Saba, M. Cadelano, D. Marongiu, F. Chen, V. Sarritzu, N. Sestu, C. Figus, M. Aresti, R. Piras, A. Geddo Lehmann, C. Cannas, A. Musinu, F. Quochi, A. Mura, and G. Bongiovanni, Correlated electron-hole plasma in organometal perovskites, Nature Communications 5, 1 (2014). 
[30] A. Kanevce, D. H. Levi, and D. Kuciauskas, The role of drift, diffusion, and recombination in time-resolved photoluminescence of CdTe solar cells determined through numerical simulation, Progress in Photovoltaics: Research and Applications 22, 1138 (2014).

[31] P. Würfel, Physics of Solar Cells (Wiley, 2005).

[32] W. Shockley and W. T. Read, Statistics of the recombinations of holes and electrons, Physical Review 87, 835 (1952).

[33] R. N. Hall, Electron-hole recombination in germanium [21] (1952).

[34] , see Supplemental Material at [URL will be inserted by publisher for additional theoretical and experimental details.

[35] R. Brenes, M. Laitz, J. Jean, D. W. deQuilettes, and V. Bulovi \ifmmode \acutec \else ć \fi, Benefit from Photon Recycling at the Maximum-Power Point of State-of-the-Art Perovskite Solar Cells, Phys. Rev. Applied 12, 14017 (2019).

[36] L. M. Pazos-Outón, M. Szumilo, R. Lamboll, J. M. Richter, M. Crespo-Quesada, M. AbdiJalebi, H. J. Beeson, M. Vrućinić, M. Alsari, H. J. Snaith, B. Ehrler, R. H. Friend, and F. Deschler, Photon recycling in lead iodide perovskite solar cells, Science 351, 1430 (2016).

[37] J. M. Richter, M. Abdi-Jalebi, A. Sadhanala, M. Tabachnyk, J. P. Rivett, L. M. Pazos-Outón, K. C. Gödel, M. Price, F. Deschler, and R. H. Friend, Enhancing photoluminescence yields in lead halide perovskites by photon recycling and light out-coupling, Nature Communications 7, 1 (2016).

[38] M. Ansari-Rad and J. Bisquert, Insight into Photon Recycling in Perovskite Semiconductors from the Concept of Photon Diffusion, Physical Review Applied 10, 034062 (2018).

[39] T. Handa, D. M. Tex, A. Shimazaki, A. Wakamiya, and Y. Kanemitsu, Charge Injection Mechanism at Heterointerfaces in CH3NH3PbI3 Perovskite Solar Cells Revealed by Simultaneous Time-Resolved Photoluminescence and Photocurrent Measurements, Journal of Physical Chemistry Letters 8, 954 (2017).

[40] J. Haddad, B. Krogmeier, B. Klingebiel, L. Krückemeier, S. Melhem, Z. Liu, J. Hüpkes, S. Mathur, and T. Kirchartz, Analyzing Interface Recombination in Lead-Halide Perovskite Solar Cells with Organic and Inorganic Hole-Transport Layers, Advanced Materials Interfaces 7, $2000366(2020)$.

[41] D. Głowienka, D. Zhang, F. Di Giacomo, M. Najafi, S. Veenstra, J. Szmytkowski, and Y. Galagan, Role of surface recombination in perovskite solar cells at the interface of 
HTL/CH3NH3PbI3, Nano Energy 67, 104186 (2020).

[42] H. J. Lin, S. Cacovich, A. Rebai, J. Rousset, and C. Longeaud, Influence of Environment and Light-Stress on the Optoelectronic Properties of Triple-Cation Perovskite Thin Films, ACS Applied Materials and Interfaces 12, 19495 (2020).

[43] J. Holovský, S. De Wolf, J. Werner, Z. Remeš, M. Müller, N. Neykova, M. Ledinský, L. Černá, P. Hrzina, P. Löper, B. Niesen, and C. Ballif, Photocurrent Spectroscopy of Perovskite Layers and Solar Cells: A Sensitive Probe of Material Degradation, Journal of Physical Chemistry Letters 8, 838 (2017).

[44] P. Löper, M. Stuckelberger, B. Niesen, J. Werner, M. Filipič, S. J. Moon, J. H. Yum, M. Topič, S. De Wolf, and C. Ballif, Complex refractive index spectra of CH3NH3PbI3 perovskite thin films determined by spectroscopic ellipsometry and spectrophotometry, Journal of Physical Chemistry Letters 6, 66 (2015).

[45] E. V. Péan, C. S. De Castro, and M. L. Davies, Shining a light on the photoluminescence behaviour of methylammonium lead iodide perovskite: investigating the competing photobrightening and photodarkening processes, Materials Letters 243, 191 (2019).

[46] S. Feldmann, S. Macpherson, S. P. Senanayak, M. Abdi-Jalebi, J. P. H. Rivett, G. Nan, G. D. Tainter, T. A. S. Doherty, K. Frohna, E. Ringe, R. H. Friend, H. Sirringhaus, M. Saliba, D. Beljonne, S. D. Stranks, and F. Deschler, Photodoping through local charge carrier accumulation in alloyed hybrid perovskites for highly efficient luminescence, Nature Photonics 2019 14:2 14, 123 (2019). 